\title{
Elastic Behavior of Zirconia under Ramp Compression
}

\author{
Binqiang Luo*, Jianjun Mo, Guiji Wang, Fuli Tan, Jianheng Zhao, and Chengwei Sun \\ Institute of Fluid Physics, CAEP, Mianyang 621900, China
}

\begin{abstract}
Dynamic properties of single crystal cubic zirconia were investigated under magnetically driven ramp wave compression on pulsed power generator CQ-4. Photonic Doppler Velocimeter (PDV) was employed to measure the free surface velocities of stepped samples. The elastic particle velocity and Ramp Elastic Limit $(R E L)$ of $<100>$ cubic zirconia under ramp wave compression are $475 \mathrm{~m} / \mathrm{s}$ and $25.4 \mathrm{GPa}$ respectively, which are much higher than shock results reported in literature. The $<110>$ cubic zirconia was loaded to maximum particle velocity $460 \mathrm{~m} / \mathrm{s}$ and pressure $20.8 \mathrm{GPa}$ in elastic region, but no distinct elasticplastic transition was observed. Inverse characteristic method was used to process the velocity data. The results show that the Lagrangian sound speed increases linearly with particle velocity in elastic region, and there exists distinct orientation effects.
\end{abstract}

\section{Introduction}

Single crystal cubic zirconia is a potentially useful window for dynamic compression research due to its transparent in $400 \mathrm{~nm}-5000 \mathrm{~nm}$ spectrum range [1] and relative high mechanical impedance and hardness. Previous shock compression experiments show that single crystal cubic zirconia has obvious anisotropy, and its Hugoniot Elastic Limit (HEL) stresses parallel to the $<100>$ and $<110>$ axes are approximately $14 \mathrm{GPa}$ and 25 $\mathrm{GPa}$ respectively [2]. Symmetric impact experiments conducted by Dolan [3] show that in elastic region the shock velocity of $\langle 100>$ cubic zirconia increases linearly with particle velocity, with a slope of 1.40 and intercept of $8.279 \mathrm{~km} / \mathrm{s}$. with different amount of doped yttrium, initial properties of zirconia such as density and ambient longitudinal wave speed change slightly.

Despite it has been used as window material in ramp compress-shear loading experiments [4, 5], there are seldom ramp compression results of single crystal cubic zirconia. In this paper, we report mechanical results of $<100>$ and $<110>$ single crystal cubic zirconia to give optimized and critical design when it is used as window material.

\section{Experiments}

Magnetically driven ramp compression experiments of $<100>$ and $<110>$ single crystal cubic zirconia were conducted on CQ-4 device to investigate the elastic behaviours and yield properties in different orientations. The principle of magnetically driven ramp compression can be found elsewhere [6]. The sketch map of target is shown in Fig. 1, four samples arrangement is used in experiments. Aluminum stripe lines conductors with $8 \mathrm{~mm}$ width were used as electrode, and zirconia samples were glued on the bottom of counter bore in electrode. A Photon Doppler Velocimeter (PDV) was employed to measure the free surface velocity of cubic zirconia samples, and the velocity profiles are shown in Fig. 2.

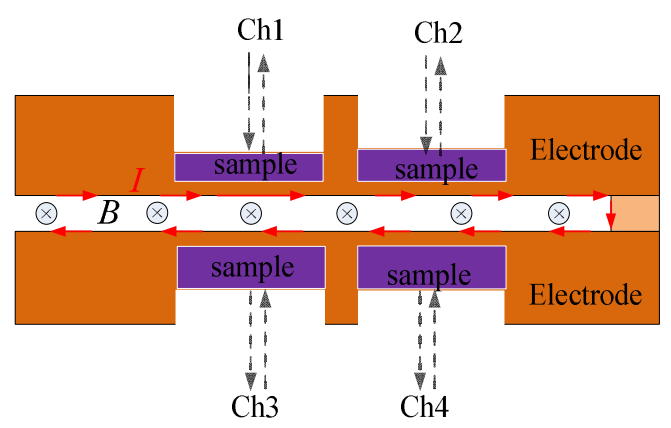

Fig. 1. Sketch map of target region.

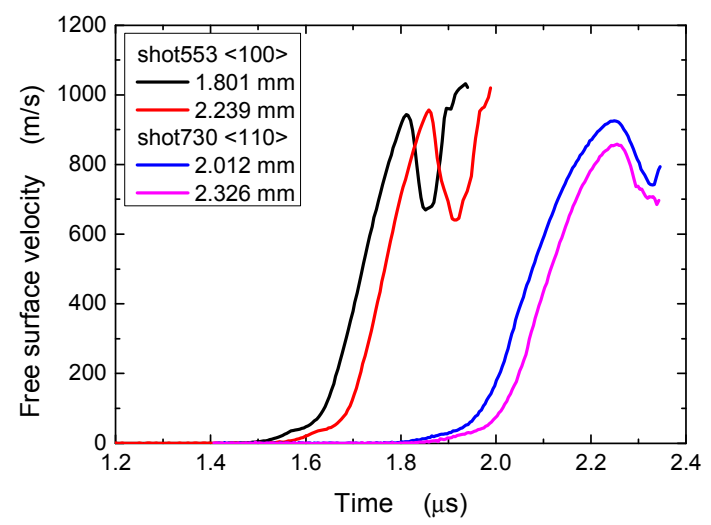

Fig. 2. Free surface velocity profiles of single crystal cubic zirconia under ramp compression.

Corresponding author: bqluoo@caep.cn 


\section{Results and discussion}

Under ramp compression, the elastic-plastic transition velocity in $<100>$ orientation is identified by the abrupt drop after elastic loading, and the amplitude is $950 \mathrm{~m} / \mathrm{s}$. In $<110>$ orientation, peak velocity of elastic loading followed by smooth drop means that rarefaction wave affected the loading process, indicating elastic-plastic transition velocity in $\langle 110\rangle$ orientation is above 920 $\mathrm{m} / \mathrm{s}$. Particle velocity at ramp elastic limit (REL) in $<100>$ orientation is $475 \mathrm{~m} / \mathrm{s}$, which is much higher than the value at HEL $270 \mathrm{~m} / \mathrm{s}$ measured by Tsutomu Mashimo $^{2}$. However, the particle velocity at REL in $<110>$ orientation (above $460 \mathrm{~m} / \mathrm{s}$ ) may be consistent with the value at HEL $510 \mathrm{~m} / \mathrm{s}$.

Inverse characteristic method was used to explore the Lagrangian sound speed $\left(\mathrm{C}_{\mathrm{L}}\right)$ versus particle velocity (up) relation from stepped sample velocity profiles. The results are shown in Fig. 3. In elastic region, Lagrangian sound speed of $\langle 100\rangle$ cubic zirconia increases linearly with particle velocity, with a relation of $\mathrm{C}_{\mathrm{L}}=8.26^{+}$ $3.20 \mathrm{u}_{\mathrm{p}}$. The intercept is consistent with the results reported by Dolan $8.279 \mathrm{~km} / \mathrm{s}$ [3] and Tsutomu Mashimo $8.231 \mathrm{~km} / \mathrm{s}$ [2]. The slope 3.20 is larger than twice of 1.40 reported by Dolan under shock compression [3]. In $<110>$ orientation, Lagrangian sound speed versus particle velocity have relation as $\mathrm{C}_{\mathrm{L}}=7.24+1.81 \mathrm{u}_{\mathrm{p}}$. The REL of single crystal cubic zirconia can be calculated as

$$
R E L=\rho_{0} \int_{0}^{u_{\text {ins_ep }}} C_{e l} d u
$$

where $\rho_{0}$ is initial density of material, $C_{e l}$ is elastic longitudinal sound speed, $\mathrm{u}_{\text {ins ep }}$ is elastic limit of particle velocity. With a linear relation of $\mathrm{C}_{\mathrm{L}}$ and $\mathrm{u}_{\mathrm{p}}$, calculated REL of $<100>$ and $<110>$ zirconia are $25.4 \mathrm{GPa}$ and above $20.8 \mathrm{GPa}$ respectively. The REL of $<100>$ zirconia is larger than the HEL 14GPa reported in ref [2] , while the REL of $\langle 110\rangle$ zirconia may be close to the HEL $25 \mathrm{GPa}$. The Poisson's ration of $<100>$ cubic zirconia calculated from ultrasonic data in ref [2] is 0.414 , and the strength of $\langle 100\rangle$ zirconia calculated from $\mathrm{Y}=(1-2 v) /(1-v) \mathrm{REL}$ is $7.3 \mathrm{GPa}$.

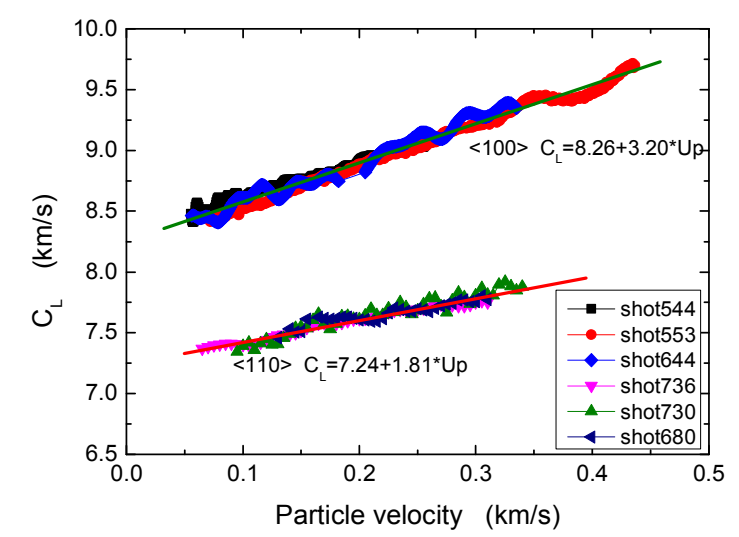

Fig. 3. Lagrangian sound speed versus particle velocity relation of $<100>$ and $<110>$ cubic zirconia.

\section{Conclusions}

Ramp compression experiments were conducted on CQ-4 device to study the elastic properties of single crystal cubic zirconia. The ramp elastic limit of $<100>$ cubic zirconia is $25.4 \mathrm{GPa}$, with elastic velocity amplitude $950 \mathrm{~m} / \mathrm{s}$ in free surface velocity. In free surface velocity of $\langle 110>$ cubic zirconia up to $920 \mathrm{~m} / \mathrm{s}$, no distinct elastic-plastic transition is observed. The ramp elastic limit of $<110>$ cubic zirconia is estimated to be above $20.8 \mathrm{GPa}$. In elastic region, the Lagrangian sound speed $\mathrm{C}_{\mathrm{L}}$ of $<100>$ and $<110>$ cubic zirconia increase linearly with particle velocity $\mathrm{u}_{\mathrm{p}}$. For $<100>$ cubic zirconia, $\mathrm{C}_{\mathrm{L}}=8.26+3.20 \mathrm{u}_{\mathrm{p}}$, for $<110>$ cubic zirconia, $\mathrm{C}_{\mathrm{L}}=7.24+1.81 \mathrm{u}_{\mathrm{p}}$.

\section{References}

1. D.L.Wood and K.Nassau, APPLIED OPTICS 21 (16), 2978-2981 (1982)

2. Tsutomu Mashimo, Akira Nakamura, Masao Kodama, Susumu Matsuzaki, Keiji Kusaba, Kiyoto Fukuoka and Y. Syono, J. Appl.Phys 77 (10), 5060-5068 (1995)

3. D. H. Dolan and T. Ao, J. Appl.Phys 93 (2), 021908 (2008)

4. C. S. Alexander, J. R. Asay and T. A. Haill, J. Appl.Phys 108 (12), 126101-126103 (2010)

5. B.Q.Luo, X.M.Chen, G.J.Wang, F.L.Tan, G.H.Chen, J.H.Zhao and C.W.Sun, International Journal of Impact Engineering 100, 56-61 (2017)

6. Guiji Wang, Binqiang Luo, Xuping Zhang, Jiangheng Zhao, Chengwei Sun,Fuli Tan, Tao Chong, Jianjun Mo,Gang Wu, and Yanhui Tao.Rev. Sci. Instrum 84 (1), 015117 (2013) 